\title{
Brain MRI Image Segmentation in View of Tumor Detection: Application to Multiple Sclerosis
}

\author{
Rabeb Mezgar ${ }^{1}$, Mohamed Ali Mahjoub ${ }^{2}$, Randa Salem ${ }^{3}$, and Abdellatif Mtibaa ${ }^{1}$ \\ ${ }^{1}$ Laboratory of Electronics and Microelectronics, Faculty of Sciences of Monastir, \\ University of Monastir, Tunisia \\ rabebmezgar@gmail.com, abdellatif.mtibaa@enim.rnu.tn \\ ${ }^{2}$ Research Unit SAGE (Advanced Systems in Electrical Engineering) Eniso, \\ University of Sousse \\ medali.mahjoub@ipeim.rnu.tn \\ ${ }^{3}$ Laboratory of interventional Radiology, \\ University of Monastir, Tunisia \\ krmranda@yahoo.fr
}

\begin{abstract}
Multiple Sclerosis (MS) is an inflammatory and demyelization disease that causes the disorder of the central nervous system. Magnetic resonance imaging (MRI) becomes the most important means for a better understanding of the disease. A variety of methods to segment these lesions are available to make the lesions detection less fastidious. So, we use a robust algorithm on EM algorithm that proposes an original detection scheme for outliers. The results obtained are very satisfactory.
\end{abstract}

Keywords: EM algorithm, Multiple sclerosis, Magnetic Resonance Imaging, Levels-Sets.

\section{Introduction}

Multiple sclerosis is in fact a disease of the central nervous system characterized by demyelization process located in the white matter resulting in the formation of plaques in relapsing multiple and successive occurring at irregular intervals whose duration is unpredictable. The cause of MS is still partially unknown. It would be rather a multifactorial disease involving primarily environmental and genetic factors. The symptoms are varied: poor balance and vision, abnormal fatigue, tremors, etc.. The major impact on knowledge of the disease and the diagnosis is magnetic resonance imaging (MRI). This also allows to monitors lesions (figure 1) over time. Faced with the increase in the amount of data for each patient, a processing system dedicated to medical image analysis of pathology must be adaptable to a changing camera settings and acquisition robust to a change the quality of images. To this end, there are several known methods: The method used to distinguish $\mathrm{T} 1$ brain tissue (white matter (MB), gray matter (GM), the cerebrospinal fluid (CSF)) and T2 modality highlights the lesions and CSF. In this context, the segmentation of brain tissue is a preliminary step in the process of detection of lesions. 


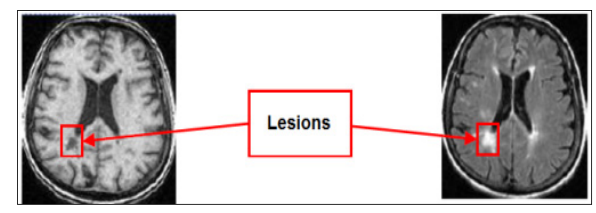

Fig. 1. MS lesions

One approach proposed for the segmentation of MRI brain is the representation of brain structures (white matter (MB), Grey Matter (GM), cerebrospinal fluid (CSF)) as components of mixture distributions for modeling the intensity as a Gaussian mixture. This model allows the modeling of the image intensities by a number of parameters. The parameter estimation is performed by the estimator of maximum likelihood (ML) using optimization methods. This classical approach is based on the ExpectationMaximization algorithm (EM) [1] and, unfortunately, is unsuitable for pathological cases characterized by atypical intensity in MRI lesions caused by MS. Changes are made to obtain the best detection results [2, 3.4].

Schroeter et al. [2] have also used a Gaussian mixture model but adding a uniform component model for the intensities of the lesions. While Van Leemput et al. [3] added a weighting that reflects the degree of "typicality" of each pixel. The data are atypical voxels (3D pixels) satisfying the following condition: their Mahalanobis distance from each component of mixture is greater than a predefined threshold. Dugas-Phocion et al. [4] introduced the calculation of the Mahalanobis distance directly in the iterations of the EM. But still, the results of these methods are still insufficient.

Our goal is therefore to provide a method then the segmentation of lesions for a given time from 3D T2-weighted FLAIR.L proposed algorithm (EM) algorithm is robust against noise and can be applied to many weights and different acquisition times. Our first contribution is the modeling of the exact multi-dimensional problem (the intensities in the image) and the robust estimation of parameters of the blend components. The second stage of our work is the extraction of Multiple Sclerosis lesions in the preamble as "outliers" of the established model. Finally, the proposed improvement is the calculation of the Mahalanobis distance of each pixel from the component mixture, which makes this data atypical of the model, after applying the optimization with the EM algorithm. The segmentation of healthy tissue and damage is then obtained.

The proposed processing chain is explained in Section 2. The following section presents the results and we conclude in Section 4.

\section{The Processing Chain}

MS lesions can be detected as voxels atypical ("Outliers") in relation to a statistical model (statistical atlas) brain images 'Normal'. In this regard, work has been performed to obtain a probabilistic atlas is used to provide information on statistical parameters of each class of brain tissue. In addition, some researchers [4] propose 
methods for obtaining a probabilistic atlas which gives the probability for each pixel to belong to a class segment. Thus, we chose to initialize the statistical parameters with values close to the values of normal patients that the algorithm is applicable to all patient image as follows: Image acquisition, extraction of brain parenchyma, parameter initialization (values of normal patient), tissue segmentation by EM algorithm and lesion extraction.

\subsection{The Brain Extraction}

In brain MRI, there is the image of the entire head. However, our goal is to focus on the part of brain tissue. So we separate them from the fat, skin... We have therefore used the geometric deformable models (Level Sets). In our case, we applied the method of implicit active contours Caselles et al. [5] with a manual initialization to segment the brain. A Level-Set is the set of points having the same function, so it's an iso contour of a function $(\mathrm{x}, \mathrm{y}, \mathrm{t})$, defined on the domain of the image. The principle a method of Level-Sets is to evolve a curve by updating a level-set function has fixed coordinates over time. This method is a method based on the contour: the gradient of the image is used to calculate the force function and the curve will be directed towards areas of high gradient. The algorithm has one parameter which is the specific term of propagation c, whose role is to push the contour to be inward or outward. Processing takes an execution time for the important initial contour being married. This treatment also requires several iterations.

\subsection{The Segmentation Method: The Algorithm Expectation-Maximization (EM)}

The algorithm (Expectation-Maximization) we present in this part was presented by Wells [6] and further developed by Van Leemput in [7, 8], and more particularly relates to the segmentation of brain MRI images. The data of the algorithm are:

$\mathrm{X}_{\mathrm{i}}$ : The intensity of the images for each voxel $\mathrm{i}$.

$\pi_{\mathrm{i}}^{\mathrm{k}}$ : The a priori probability for each voxel $\mathrm{i}$ belonging to class $\mathrm{k}$.

The results are to seek: $\left(\mu_{\mathrm{k}} \sum_{\mathrm{k}}\right)$ : Parameters of Gaussians associated with each class $Y_{\mathrm{i}}^{\mathrm{k}}$ : The posterior probability of belonging to class $\mathrm{k}$.

The a priori probability $\pi_{\mathrm{i}}^{\mathrm{k}}$ to belong to a class for each voxel $\mathrm{k}$ i can be available as an atlas or statistical probabilities. The different parameters can be estimated as follows :

$$
\begin{gathered}
\mu_{\mathrm{k}}=\frac{\sum_{\mathrm{i}=1}^{\mathrm{N}} \mathrm{x}_{\mathrm{i}} \Upsilon_{\mathrm{i}}^{\mathrm{k}}}{\sum_{\mathrm{i}=1}^{\mathrm{N}} \Upsilon_{\mathrm{i}}^{\mathrm{k}}} \\
\sum_{\mathrm{k}}=\frac{\sum_{\mathrm{i}=1}^{\mathrm{N}}\left(\mathrm{x}_{\mathrm{i}}-\mu_{\mathrm{k}}\right)\left(\mathrm{x}_{\mathrm{i}}-\mu_{\mathrm{k}}\right)^{\mathrm{T}} \Upsilon_{\mathrm{i}}^{\mathrm{k}}}{\sum_{\mathrm{i}=1}^{\mathrm{N}} \gamma_{\mathrm{i}}^{\mathrm{k}}}
\end{gathered}
$$


If, however, the labelings are known, the parameters of classes are directly calculable from the labeling. The updating of posterior probabilities is done by the following formula:

$$
\Upsilon_{i}^{k}=\frac{G_{\mu k} \sum_{k}\left(x_{i}\right) \pi_{i}^{k}}{\sum_{l=1}^{k} \pi_{i}^{k} G_{\mu l} \sum_{l}\left(x_{i}\right)}
$$

The intensity distribution of each class of tissue is approximated by a Gaussian $\mathrm{G}\left(\mu_{k}\right.$, $\Sigma_{k}$ ) average $\mu_{k}$ and covariance matrix $\Sigma_{k}$. And the reason for the use of mixtures of Gaussian modeling is the presence of different component in each voxel (idea proposed by Schroeter and Al) [9]. The probability distribution in a mixture model [10], $\mathrm{X} \mid \theta$ is defined as a weighted sum of parametric functions $\mathrm{K}$, whose set of parameters $\theta$ :

$$
P(X \mid \theta)=\sum_{k=1}^{K} p((X, Z=k) \mid \theta)=\sum_{k=1}^{K} p(Z=k) p(X \mid(Z=k, \theta))=\sum_{k=1}^{K} \pi_{k} p((X, Z=k) \mid \theta)
$$

Indeed, $\mathrm{p}(\mathrm{XI}(\mathrm{Z}=\mathrm{K}, \theta))$ is a combination of $\mathrm{K}$ Gaussian parameters $\left(\mu_{\mathrm{k}}, \sum_{\mathrm{k}}\right)$. In our model, the parameters are:

$$
\theta=\left\{\pi_{1}, \pi_{2}, \ldots, \pi_{k}, \mu_{1}, \mu_{2}, \ldots, \mu_{k}, \sum_{1}, \sum_{2}, \ldots, \sum_{k}\right\}
$$

The EM algorithm can estimate the parameters $\theta$ based on the data $\mathrm{X}$. In this model (model mixture of Gaussian), the membership of each sample to a class $\mathrm{k}$ is represented by the hidden variable Z.L estimate would be easy with this information on data labelized. The posterior probability of obtaining a labeling $\mathrm{Zi}=\mathrm{k}$ according to the parameters $\boldsymbol{\theta}_{\mathbf{k}}$ class $\mathrm{k}$, and xi is given:

$$
Y_{\mathrm{i}}^{\mathrm{k}}=\mathrm{p}\left(\mathrm{Z}_{\mathrm{i}}=\mathrm{k} \mid \mathrm{X}=\mathrm{x}_{\mathrm{i}}, \theta_{\mathrm{k}}\right)
$$

The $\Upsilon_{\mathrm{i}}^{\mathrm{k}}$ are labeling a posteriori data. The step of expectation is done using Bayes law:

$$
Y_{\mathrm{i}}^{\mathrm{k}}=\frac{\mathrm{p}\left(\mathrm{Z}_{\mathrm{i}}=\mathrm{k}\right) \mathrm{p}\left(\mathrm{X}=\mathrm{x}_{\mathrm{i}} \mid \mathrm{Z}_{\mathrm{i}}=\mathrm{k}, \theta_{\mathrm{k}}\right)}{\mathrm{p}\left(\mathrm{X}=\mathrm{x}_{\mathrm{i}}, \theta_{\mathrm{k}}\right)}=\frac{\pi_{\mathrm{k}} \mathrm{G}_{\mu(\mathrm{K}), \Sigma(\mathrm{k})}(\mathrm{x})}{\sum_{\mathrm{i}=1}^{\mathrm{K}} \pi_{\mathrm{k}} \mathrm{G}_{\mu(\mathrm{K}), \Sigma(\mathrm{k})}(\mathrm{x})}
$$

The iteration of steps 2 and 3 ends when we reach the convergence criterion that is related to the change in the log-likelihood:

$$
\left(\mathrm{L}\left(\mathrm{X} \mid \theta^{(\mathrm{t}+1)}\right)-\mathrm{L}\left(\mathrm{X} \mid \theta^{(\mathrm{t})}\right)\right) \leq \varepsilon
$$

- The space of intensities

The joint histogram is a handy tool to see the space of currents: It represents the distribution of intensities based on grayscale and Gaussian distribution. Representation is possible: the idea is to isolate the "Outliers" by the CSF to put them in a class that decomposes the image into four classes instead of three. Histogram for the difference lies in the histograms (green) of the Gaussian distribution that become four (fig. 2). 
- The images space

The result of the algorithm is a mask that represents the posteriori labeling of voxels. The EM algorithm takes as input image to segment (stage supratentorial) and the number of classes. In case $\mathrm{k}=3$, the mask has a good classification of regions and MS lesions are well defined in a whole class (fig. 3). The ownership of the T2 FLAIR provides good contrast for the detection of lesions of Multiple Sclerosis because it distinguishes the cerebra-spinal plates: only take the white lesions (hyper signal).

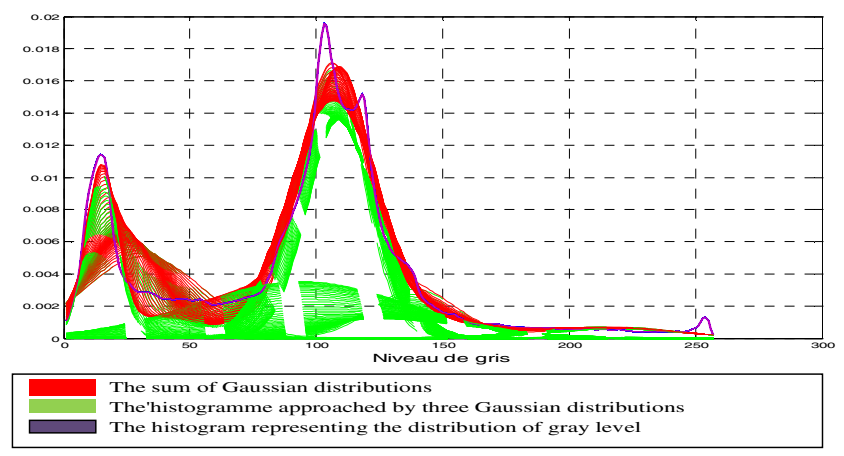

Fig. 2. Histograms representing the distributions of the intensity in the image (Case classification into 4 classes (white matter, gray matter, CSF, "Outliers")

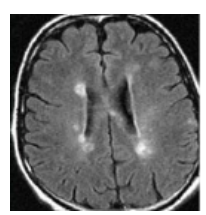

a)

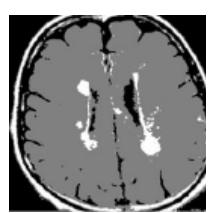

b)

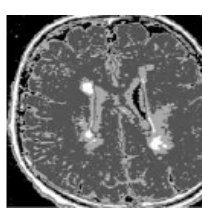

c)

Fig. 3. Segmentation results by EM algorithm a) original image b) segmentation with 3 classes c) 4 classes

- The space of statistical parameters

Table 1 shows the statistical parameters by considering an EM algorithm for 4 classes:

Table 1. Statistical parameters for 4 classes

\begin{tabular}{llll}
\hline & Mean & Variance & Probability \\
\hline Class1 & 14 & 38,7 & 0,16 \\
Class 2 & 108,2 & 156,7 & 0,42 \\
Class 3 & 93,26 & 1776,8 & 0,37 \\
Class 4 & 218,17 & 696 & 0,03 \\
\hline
\end{tabular}


We have differentiated the CSF of "outliers" where each tissue represents a whole class. In this case to distinguish between the two classes, it should be noted that the class "outliers" is often a very significant variance compared to other classes. So the class concerned in this case is the third class.

\subsection{Extracting the Lesions: The Mahalanobis Distance}

Once the data model is established by MS, we then seek to extract the "Outliers" (atypical data). For each voxel $i$ of the image, the Mahalanobis distance between the vector yi and each class $\mathrm{k}$ is:

$$
\mathrm{D}_{\mathrm{M}}(\mathrm{x})=\operatorname{sqrt}\left(\left(\mathrm{y}_{\mathrm{i}}-\mu_{\mathrm{k}}\right)^{\mathrm{T}} \Sigma_{\mathrm{k}}^{-1}\left(\mathrm{y}_{\mathrm{i}}-\mu_{\mathrm{k}}\right)\right)
$$

$\mathrm{DM}$ is the "Mahalanobis distance" from the point $\mathrm{x}$ to the mean $\mu$ of the distribution. In the implementation algorithms, we sought to determine the distance of the voxels from the voxels belonging to the third class (which contains outliers) so we looked for the difference in values of the voxels relative to the mean and variance of third Class. Voxels representing the lesions are those values above the mean and variance of the third class.

\section{Experiments}

In case the number of classes is three (MB, MG, LCR + 'Outliers') detection is not complete (Fig. 4). Indeed, there is elimination of noise but also removal of some MS lesions. The execution time is of the order of seconds. The EM algorithm takes 16.72 seconds, while the calculation of the Mahalanobis distance is 20.41 seconds.

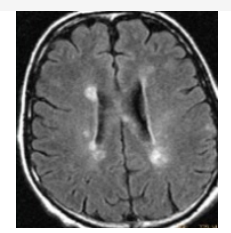

(a)

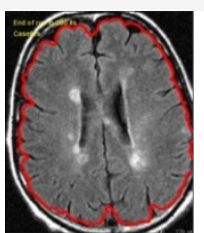

(b)

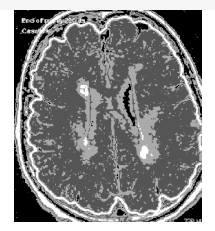

(c)

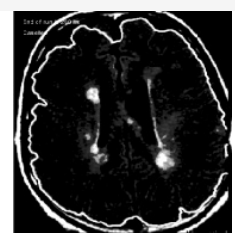

(d)

Fig. 4. Results of treatment $(k=3)$ original image b) parameters estimation $c)$ determination of brain parenchyma mask d) lesion detection

If we differentiate CSF and "outliers" we obtain more accurate results (Fig. 5). The execution time of the EM algorithm for four classes is higher 29.55 seconds, because of increasing of iterations number for parameter estimation. The time for calculating the Mahalanobis distance is almost identical 20.99 seconds.

Our main objective is to obtain a better estimation of the parameters of classes, not just to get a good labeling for the detection of lesions. Our processing chain has been very acceptable detection of MS in a short execution time for $\mathrm{k}=4$ (Fig. 5). We managed to obtain clinical validation by a doctor, we have to validate our work by a quantitative assessment. 


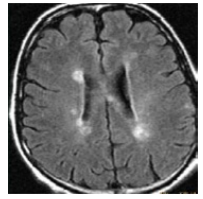

a)

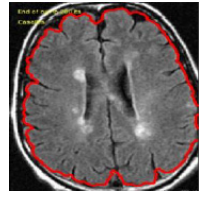

b)

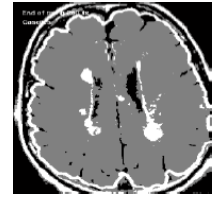

c)

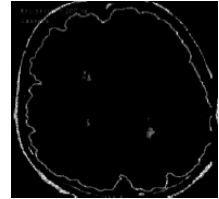

d)

Fig. 5. Results of treatment $(k=4)$ Original image b) parameters estimation c) determination of brain parenchyma mask d) lesion detection

\subsection{Evaluation and Validation of Results}

Our algorithm is characterized by a very short execution time and the response is almost instantaneous. The evaluation parameters are:

- Sensitivity (Se): if the area is detected a lesion, the probability of being detected as lesions.

- Specificity (Sp): If the detected area is healthy, it is likely detected as sound.

- Positive predictive value (PPV): If the test is positive probability that the area is actually detected a lesion.

- Negative predictive value (NPV): If the test is negative, the probability that the area detected is actually healthy.

- TP(True Positive):detected as hyperintense pathological lesions.

- TN(True Negative):non pathological hyperintensities detected as such.

- FP(False Positive):non pathological hyperintensities(artifacts,noise..) detected as lesions.

- FN(False Negative):hyperintense pathological ignored by the processing chain.

Table 2. Quality assessment of a drug test

\begin{tabular}{llll}
\hline & Lesion & healthy & quantifier \\
\hline positive test & $\mathrm{TP}$ & $\mathrm{FP}$ & $\mathrm{V}_{\mathrm{PP}}$ \\
negative test & $\mathrm{FN}$ & $\mathrm{TN}$ & $\mathrm{V}_{\mathrm{PN}}$ \\
quantifier & $\mathrm{Se}$ & $\mathrm{Sp}$ & ---- \\
\hline
\end{tabular}

The test is applied on subjects including sick or not lesion volume (column), it can be positive or negative (line). Good sensitivity is a sign that a large majority of lesions are detected as good damage-detection - while a good specificity suggests that few healthy areas are detected as injury - no false alarms. When:

$$
S e=\frac{\mathrm{VP}}{\mathrm{VP}+\mathrm{FN}} \quad ; S p=\frac{\mathrm{VN}}{\mathrm{VN}+\mathrm{FP}} \quad ; V_{P P}=\frac{\mathrm{VP}}{\mathrm{VP}+\mathrm{FP}} \quad ; V_{P N}=\frac{V N}{V N+F N} \quad ;
$$

If the test is perfect, there will be no false positive or false negative. In practice, when a doctor sees the results of detection system, positive or negative, the question implies, what is the probability that the detected area is really an injury, knowing that 
the review gave a positive (or negative ) ? What are the predictive values that correspond to the concerns of doctors, and they might seem evaluation parameters the most significant. But really, it is the specificity and sensitivity that are most often used to assess the additional tests.

\subsection{Quantitative Evaluation of Segmentation of MS Lesions}

We validated our processing chain on T2 FLAIR MRI images of 15 patients by Multiple Sclerosis. The results of the chain of detection vary from case to case. Several factors affect the detection of lesions: the type of lesions, the presence of flow artifacts (false positives) and the presence of "black holes" (false negatives). The result of detection chain of MS lesions is presented in Fig. 6 concerning the first patient.

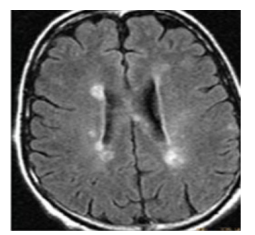

(a)

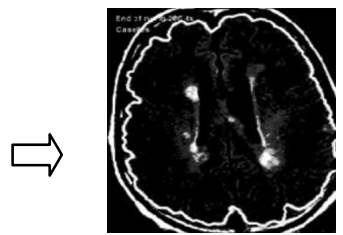

(b)

Fig. 6. Results of treatment of first patient original image b) lesion detection

The results of detection of per ventricular lesions are acceptable in terms of sensitivity and specificity (Table 3). The majority of lesions detected are well except that the horns of the ventricles and the space between the two ventricles often suffer from artifacts in T2 FLAIR flow, causing false positives.

Table 3. Evaluation of quality detection

\begin{tabular}{llll}
\hline & Lesion & healthy & quantifier \\
\hline positive test & 5 & 2 & 0.714 \\
negative test & 0 & 0 & 0 \\
quantifier & 1 & 0 & ---- \\
\hline
\end{tabular}

\subsection{The ROC (Receiver Operating Characteristics)}

When a review provides the results of the continuous type, it determines the best detection limit among the pathological values. The ideal would be to obtain a sensitivity and specificity equal to 1 . This is usually not possible, and we must try to obtain the highest values for these parameters, knowing that they vary in opposite directions. For a good choice, we make a graphical tool, the ROC curve (Fig. 7). It is the plot of the values of the sensitivity versus $1 \mathrm{Se}-\mathrm{Sp}$ Just then look for the point of the curve that is closest to the point coordinate $(\mathrm{Se}=1 ; 1-\mathrm{Sp}=0)$. 


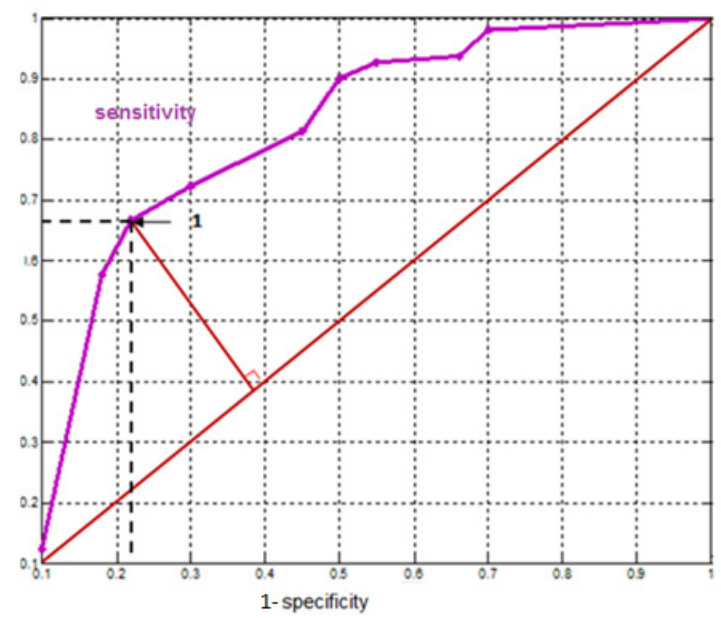

Fig. 7. ROC curve for 1'examen of 15 patients

In this figure, the curve is a good diagnostic test for which we can simultaneously obtain high values of sensitivity and specificity. The threshold is the desired coordinates satisfying a maximum value of sensitivity and specificity (in the ideal case: $1,1-\mathrm{Se}=\mathrm{Sp}=0$ ). Example of choice of an optimal threshold value with a ROC curve: a threshold value (sensitivity and specificity $=0.723=0.8$ ) is obtained by finding the point on the curve farthest from the diagonal. The area under the curve associated with greater than $0.5(\approx 0.75)$ so this is a test more or less perfect (a perfect test, the area under the curve is associated with 1$)$. There are on average VP $=7.6$ true positives and FP $=1.468 .92$ false positives among the lesions seen by physicians. Although the number of false positives is not important compared to true positives but there is always a degradation factor because in the clinical examination should distinguish the lesions in order to properly assess the influence of treatment over several flares.

Table 4. Detection: Calculation of quantifiers

\begin{tabular}{|c|c|c|c|}
\hline & $\begin{array}{c}\text { Labeled as } \\
\text { lesions }\end{array}$ & $\begin{array}{c}\text { Labeled } \\
\text { as healthy }\end{array}$ & Quantifiers \\
\hline detected as lesions & 7,6 & 1,46 & $\operatorname{Vpp}(\operatorname{vox})=0,83$ \\
\hline Detected as healthy & 1,32 & ---- & ----- \\
\hline Quantifiers & $\operatorname{Se}(\operatorname{vox})=0,85$ & & \\
\hline
\end{tabular}

In view of results, the segmentation system can be described as sensitive: $\mathrm{Se}=$ $85 \%$ of lesions are detected. And we see that $27 \%$ (1-Vpp) lesions machines are false positives. For the detection of lesions of multiple sclerosis, we achieve good sensitivity. As part of a diagnostic aid, for example, a false negative lesion that is not detected by the chain, must be sought by the doctor, which costs a lot of time. On the other hand, a false positive can be corrected afterwards: a manual correction by an expert may be added or a priori by the introduction of a probabilistic atlas. 


\section{Conclusion}

This work is included in the development of approaches to help specialists to make accurate decisions in monitoring patients with lesions in the white matter. Our contribution is the development of a processing chain which aims to detect lesions as "Multiple Sclerosis". To achieve this, the segmentation of MRI brain is the essential step in the detection process. Thus, we proposed an efficient algorithm for segmentation of MRI T2-FLAIR based on EM algorithm. The convergence of such iterative scheme has been demonstrated. The initialization of parameters is done randomly; in order to improve our results we initialized them using a brain atlas flunked our data to rigidly. To improve the detection results of the processing chain, it is necessary to eliminate these false positives. In the literature, approaches are proposed to address this problem that are moving towards the introduction of a priori knowledge by using the statistical atlas, the integration of certain processes to improve the visual quality of the segmentation. We can think about different methods of segmentation such as hidden Markov chain prior to our detection to include constraints on the neighborhood. We can also think about separating the outliers by searching for a method to determine a threshold that can differentiate the "Outliers" in cerebrospinal fluid.

\section{References}

1. Dempster, A., Laird, N., Rubin, D.: Maximum likelihood from incomplete data via the EM algorithm. Journal of the Royal Statistical Society 39(1), 1-38 (1977)

2. Schroeter, P., Vesin, J.M., Langenberger, T., Meuli, R.: Robust Parameter Estimation of Intensity Distributions for Brain Magnetic Resonance Images. IEEE Transactions on Medical Imaging 17(2), 172-186 (1998)

3. Van Leemput, K., Maes, F., Vandermeulen, D., Colchester, A., Suetens, P.: Automated segmentation of multiple sclerosis lesions by model outlier detection. IEEE Transactions on Medical Imaging 20(8), 677-688 (2001)

4. Dugas-Phocion, G., González, M.A., Lebrun, C., Chanalet, S., Bensa, C., Malandain, G., Ayache, N.: Hierarchical Segmentation of Multiple Sclerosis Lesions in Multi-sequence MRI. In: ISBI 2004, Arlington, USA (April 2004)

5. Caselles, V., Kimmel, R., Sapiro, G.: Geodesic active contours. Int. J. of Computer Vision 22, 61-79 (1997)

6. Wells III, W.M., Grimson, W.E.L., Kikinis, R., Jolesz, F.A.: Adaptive Segmentation of MRI Data. In: Ayache, N. (ed.) CVRMed 1995. LNCS, vol. 905, pp. 59-69. Springer, Heidelberg (1995)

7. Van Leemput, K., Maes, F., Vandermeulen, D., Suetens, P.: Automated model-based bias field correction of MR images of the brain. IEEE Transactions on Medical Imaging, 885-896 (October 1999)

8. Van Leemput, K., Maes, F., Vandermeulen, D., Suetens, P.: Automated model-based tissue classification of MR images of the brain. IEEE Transactions on Medical Imaging 897-908 (October 1999) 
9. Dugas-Phocion, G.: Segmentation d'IRM Cérébrales Multi-Séquences et Application à la Sclérose en Plaques, Thèse de doctorat (2006)

10. McLachlan, G., Peel, D.: Finite mixture models. Wiley Series in Probability and Statistics (2000)

11. Flandin, G.: Utilisation d'informations géométriques pour l'analyse statistique des données d'IRM fonctionnelle. PhD thesis, Université de Nice-Sophia Antipolis (April 2004)

12. Mahjoub, M.A., Kalti, K.: Image Segmentation by Adaptive Distance Based on EM Algorithm. In: IJACSA, pp. 19-24 (2010) 